\title{
Kleinskalige, multidirektionale Transportmodule für den Einsatz in der Intralogistik
}

\author{
Prof. Dr.-Ing. Ludger Overmeyer \\ Institut für Transport- und Automatisierungstechnik \\ Gottfried Wilhelm Leibniz Universität Hannover \\ Dipl.-Ing. Kai Ventz \\ Transnorm System GmbH, Harsum \\ Dipl.-Ing. Sascha Falkenberg \\ Institut für Transport- und Automatisierungstechnik \\ Gottfried Wilhelm Leibniz Universität Hannover
}

\section{Zusammenfassung:}

Der Beitrag betrachtet die Möglichkeiten einer innovativen Gestaltung von Intralogistik durch Kopplung kleinskaliger Systeme. Intralogistische Systeme sind heute sehr anwendungsspezifisch geprägt, welches speziell zugeschnittene Lösungen und damit kleine Stückzahlen bei der Produktion erfordert.

Die Steuerung dieser Systeme erfolgt überwiegend zentral. Um dem Trend nach verkürzten Produktlebenszyklen nachzukommen und individuelle materialflußtechnische Lösungen anbieten zu können, ist eine größere Flexibilität der Intralogistik-Systeme erforderlich. Gleichartige, kleinskalige Module mit eingeprägter Intelligenz und standardisierten Schnittstellen bieten hier einen interessanten Lösungsansatz.

Es werden die Anforderungen und Möglichkeiten untersucht, ein kleinskaliges fördertechnisches Modul zum Aufbau eines flexiblen intralogistischen Systems zu entwickeln. Eine wesentliche Anforderung an ein fördertechnisches Verfahren für ein kleinskaliges Modul ist größtmögliche Flexibilität, um mit den Modulen individuelle fördertechnische Aufgaben lösen zu können. Die Module müssen daher möglichst viele Funktionalitäten in sich vereinen, um neben der reinen Transportaufgabe beispielsweise auch Ausschleusungen, Richtungsänderungen und individuelle Zielfindung überall in System zu ermöglichen. Darüber hinaus soll eine Erweiterung des Systems in allen Richtungen in der Ebene möglich sein. Es werden dabei Lösungsansätze für ein geeignetes fördertechnisches Verfahren auf basierender Technik wie z.B. Gurt- und Rollenfördertechnik betrachtet und methodisch nach neuen Lösungen gesucht. Die Weiterentwicklung der Schwenkrollentechnik und einige neue Ansätze wie der Einsatz von Schwenkscheiben sind vielversprechende Lösungen. Mit dieser flexiblen mechanischen Basis lassen sich kleinskalige fördertechnische Module konstruieren, die dann zu selbststeuernden Elementen weiterentwickelt werden.

\section{Einleitung}

Die innerbetriebliche Materialflußtechnik als Teil der Intralogistik-Branche bietet nahezu sämtlichen Wirtschaftszweigen enormes Rationalisierungspotential [ARN 06, WOL 07]. Die Anforderungen an ein Materialflußsystem werden dabei immer komplexer, aus mechanischer Sicht das zu bewegende Fördergut betreffend so wie die Funktionalität und die Anlagensteuerung betreffend. Unterschiedlichstes Fördergut von kleinsten unverpackten Teilen bis zu großen Kartons und Behältern, die gemeinsam ein Materialflußsystem nutzen, bedeuten gegenläufige Anforderungen an das System.

Funktionalitäten sind speziell auf den Anwendungsfall zuzuschneiden, vom reinen Transport bis zu komplexen Sortier- und Ausschleusfunktionen z.B. zur vollautomatischen Lagerbestückung oder Warenkommissionierung. Die heute üblichen Materialflußsysteme sind weitestgehend zentral gesteuert, wie beispielsweise über eine Speicherprogrammierbare Steuerung (SPS). Die Position des Fördergutes wird z.B. über optische Sensorik wie Lichtschranken ermittelt und ggf. über Wegeverfolgung weiter errechnet, um dementsprechend Sortier- und Ausschleusvorgänge einzuleiten. Mechanisch besitzen sie eine definierte Ausprägung, die weitgehend festliegt und damit sind auch Funktionalitäten, wie z.B. das Ausschleusen, im System lokal gebunden. Solche Funktionalitäten werden in der Regel auf das größte im System zu bewegende Fördergut ausgelegt und bedeuten für kleineres Fördergut Leistungsverlust. Veränderungen sind nur mit relativ großem Aufwand möglich. Besonders Neuverkabelung und Neuprogrammierung der zentralen Steuerung sind ausgesprochen zeit- und kostenintensiv. Eine zukunftsweisende, fördertechnische Lösung muß mit implementierter Intelligenz individuelle komplexe Transportaufgaben erfüllen und dabei flexibel für Veränderungen und Anpassungen bei gleichzeitig möglichst geringem Investitionsaufwand sein. 
Gleichartige, kleinskalige Förderkomponenten mit integrierter Steuerung und Informationsverarbeitung bieten einen Lösungsansatz. Einzelne Module lösen im Zusammenschluß komplexe Materialflußaufgaben in der Ebene und versprechen durch ihre hohe Gleichartigkeit bei vergleichsweise kleinen Abmessungen Kostenvorteile. Denn die Vorteile der Gleichschartigkeit lassen sich zum einen produktionstechnisch durch erhöhte Stückzahlen gleicher Elemente und damit verbundenen sinken Produktionskosten nutzen, zum anderen entstehen standardisierte Schnittstellen, die den Planungs- und Installationsaufwand erheblich senken.

Eine wesentliche Rolle spielen beim Betrieb einer intralogistischen Anlage Wartung, Reparatur und funktionale Flexibilität, bis dato durch Austausch spezifischer Elemente und Montagearbeiten vor Ort realisiert. Preiswerte kleinskalige Module erlauben dagegen einen einfachen Austausch kompletter Module ohne aufwendige Montage- und Justagearbeiten vor Ort.

Ein wichtiger Aspekt der Modularisierung ist die Schaffung einzelner dezentraler Intelligenzen statt einer zentralen, übergeordneten und steuernden Hierarchie, da das Potential der Modularität mit autonomen Einheiten am weitesten genutzt werden kann. Ansätze dezentraler modularer, Komponenten sind realisiert und beschrieben in [DEM 08, TRA 08]. Dezentrale Strukturen wie das Internet und Verhaltensmuster aus der Natur wie das Verhalten von Gruppen dienen bei der Entwicklung einer Steuerung als Modell [OVE 07]. Aus den einzelnen Interaktionen entsteht ein nutzbringendes Ganzes. Als Beispiel seien Ameisenkulturen genannt. Allen gemein ist die fehlende Hierarchie und die hohe Robustheit bzw. Ausfallsicherheit. Die Intelligenz oder steuernde Logik ist auf die Teilnehmer des Systems verteilt und das Gesamtsystem ist nahezu beliebig erweiterbar.

Selbststeuernde fördertechnische Prozessen sind u.a. in [HOM 06] beschrieben.

Das Internet der Dinge auch unter Anwendung der RFID-Technologie [SEI 05, FLE 05, OVE 05] geht von einer dezentralen, auf fördertechnische Elemente verteilten Intelligenz aus. Das Fördergut selbst kann durch Kommunikation mit dem Fördersystem seinen Weg beeinflussen, beispielsweise durch RFID-Etiketten, die von Förderelementen erkannt und ausgelesen werden und das Förderelement steuern. Die Streckenführung kann damit flexibel gestaltet werden. Standardisierte Schnittstellen an gleichartigen Modulen sind notwendig und senken darüber hinaus den Planungs- und Installationsaufwand erheblich.

Es ergibt sich die Aufgabe, kleinskalige Materialfluß-Module zu entwickeln, die sich bei hohen Stückzahlen kostengünstig produzieren lassen. Die Kopplung mehrerer Module läßt durch Interaktions-Wirkprinzipien, wie z.B. Vernetzung, eine fördertechnische Funktionalität entstehen (Abbildung. 1). Besonderes Interesse gilt bei der Umsetzung des Modularitätsprinzips den Förderelementen der unteren Gewichtsklasse (GK1, bis 50 kg) [DUB 05]. In dieser Klasse erscheint der Modularitätsansatz in Verbindung mit einer Verkleinerung der Förderelemente vielversprechend.

\section{Anforderungen an ein kleinskaliges Transport-Modul}

Es ist ein kleinskaliges Modul zu entwickeln, das sich in großen Stückzahlen kostengünstig produzieren lässt und durch dessen Kopplung mit vielen gleichartigen Modulen ein fördertechnisches System entsteht. Teilaufgaben betreffen die Mechanik, den Antrieb und die Steuerung sowie die Kommunikation. Die Frage nach der Funktionalität eines Grundmoduls hängt dabei stark von dem Funktionsanspruch des Gesamtsystems ab. Denkbar sind Grundmodule, die beispielsweise als Gurtförderer-Miniaturen mit Förderfunktion in Hauptförderrichtung $\mathrm{x}$ und entsprechend implementierter Steuerungslogik und den dazugehörigen Schnittstellen zur Kopplung untereinander ausgeführt sind. Das modular aufgebaute System kann alle erdenklichen Funktionen wie z.B. Akkumulieren in Hauptförderrichtung $\mathrm{x}$ leisten. Für Funktionalitäten in $\mathrm{y}$-Richtung, wie Ausschleusungen und Sortierfunktionen, sind weitere oder zusätzliche Module notwendig, die allerdings die Funktionalität im System lokal binden. Module mit der Möglichkeit des Materialflusses in x- und y-Richtung sind vom Grundaufbau aufwendiger, der modulare Gedanke wird jedoch konsequenter abgebildet. Ausschleusund Sortierfunktionen sind flexibel überall im System möglich.

Je nach funktionalem Anspruch des Gesamtsystems gibt es mehrere Möglichkeiten ein modulares System aufzubauen. Betrachtet wird zunächst die mechanische Funktionalität. Gegliedert wird in drei Ebenen:

1. Module mit einfachem Funktionsumfang, z.B. Gurt- oder Rollenfördererminiaturen

2. Module mit einfachem Funktionsumfang und zusätzliche Module mit erweitertem Funktionsumfang nach Bedarf, d.h. ergänzende Module die Ausschleus- und Sortierfunktionen übernehmen

3. Ausschließlich Module mit erweitertem Funktionsumfang, d.h. omnidirektionale Module, die umfangreiche fördertechnische Aufgaben in der Ebene übernehmen

Die größtmögliche Flexibilität bieten Module mit omnidirektionaler Funktionalität. Tabelle 1 zeigt einen Vergleich der Konzepte mit unterschiedlichem mechanischem Funktionsumfang der Module: 


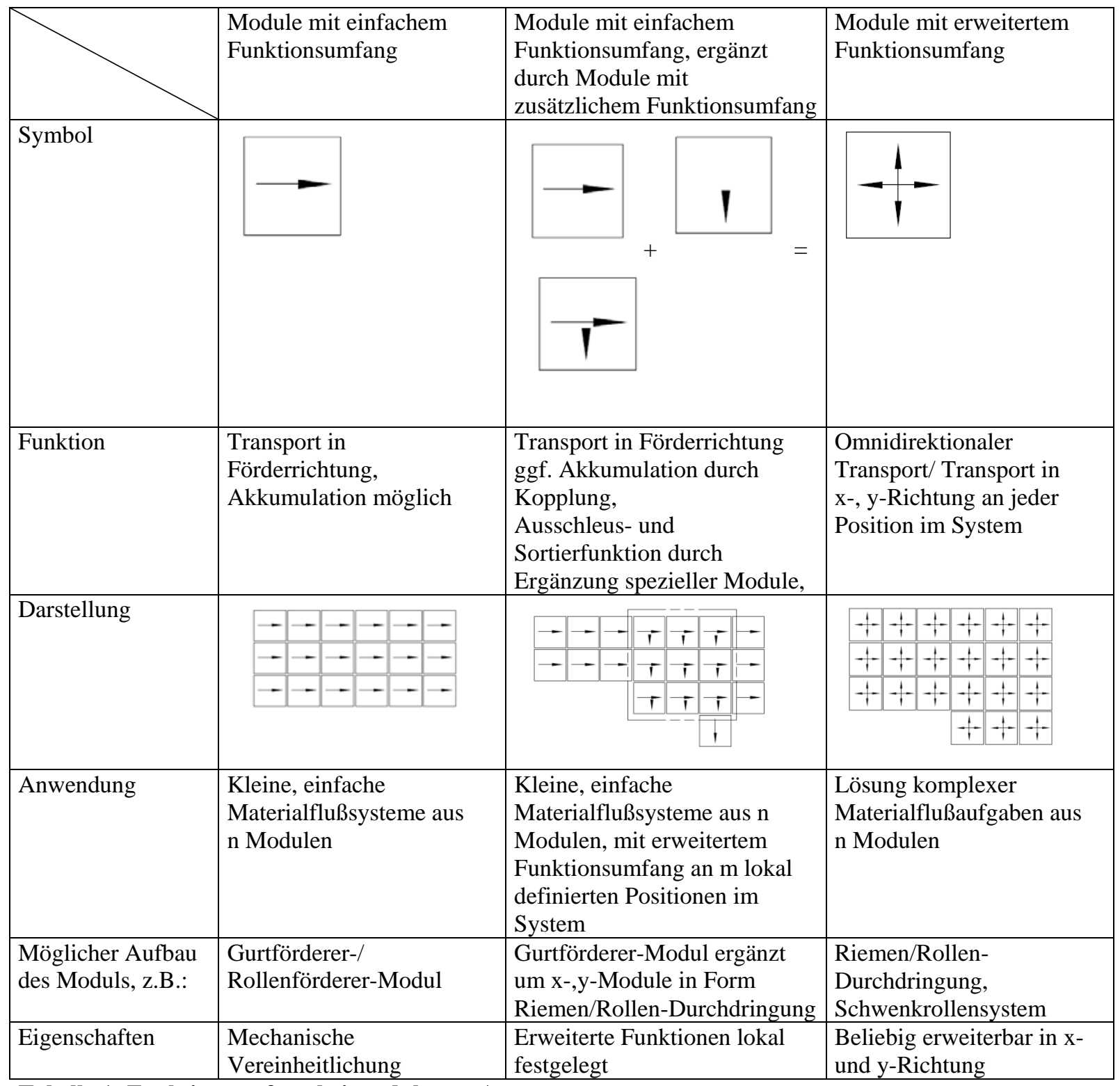

\section{Tabelle 1: Funktionsumfang bei modularem Ansatz}

Das Grundmodul muß eine hohe Verträglichkeit mit dem Fördergut besitzen, insbesondere an den Übergabestellen. Ein hohes Maß an Kleinskaligkeit bedeutet eine höhere Anzahl von Systemgrenzen. Von Bedeutung ist der Zusammenhang von Bauteilgröße und anzustrebender Fördergeschwindigkeit. Übliche Geschwindigkeiten liegen bei intralogistischen Anlagen für den reinen Transport bei Gurt und Rollenfördersystemen im Bereich von 30 bis $120 \mathrm{~m} / \mathrm{min}$. Für Funktionalitäten wie Sortieren, z.B. Anschlußstellen an Sortern, sind bereits Geschwindigkeiten von über $120 \mathrm{~m} / \mathrm{min}$ realisiert worden.

Zur Steuerung der fördertechnischen Aufgaben ist die Positionsabfrage und Identifizierung der Transporteinheiten notwendig. Die Erfassung z.B. mittels optischer Sensorik ist von der Materialbeschaffenheit der Transporteinheit unabhängig und damit vorteilhaft im Vergleich zu kapazitiven oder magnetischen Verfahren. Die Gestaltung der optischen Erfassung der Transporteinheit auf den Fördermodulen richtet sich nach der Größe und Gestalt der Transporteinheit bezogen auf die Modulgröße und auf die Anzahl der steuerungstechnisch zusammengefassten Module.

In der Fördertechnik haben sich optische Näherungsschalter bewährt, die es bereits in sehr kleiner Bauform gibt. Interessant sind auch Lösungen mit Reflextastern in Kombination mit Lichtleitern. Die sehr klein bauende Lichtleiteroptik kann platzsparend am Meßort untergebracht werden, die Meßelektronik kann außerhalb der Meßstelle plaziert werden. Auch neue Verfahren zur Integration von optischen Leitern zur Datenübertragung sind nutzbringend einsetzbar [FAH 08]. 
Zur Implementierung einer eigenen Intelligenz und erweiterter Funktionalität ist es notwendig, jedes Modul mit Sensorik und Steuerungslogik auszustatten. Die modulare Bauweise der Förderelemente soll eine Erweiterbarkeit in allen Richtungen ermöglichen. Reflexionslichtschranken und Einweglichtschranken haben den Vorteil der hohen Zuverlässigkeit bei geringen Kosten. Nachteil ist, daß nur Bereiche über die gesamte Breite oder Länge einer Fördereinheit abgefragt werden können. Für erweiterte Funktionalitäten ist es erforderlich, kleinere Bereiche abzufragen, falls sich beispielsweise Transporteinheiten hinter- und nebeneinander auf einer Fördererstrecke mit Sortierfunktion befinden. Eine modulweise optische Abfrage eines zu transportierenden Objektes ist bei Integration der Sensorik in das Modul nur durch die Positionierung der Sensorik unterhalb des Moduls, d.h. Erkennung der Transporteinheiten von unten möglich. Nachteilig ist die mögliche Verschmutzung der Optik im Betrieb.

\section{Modulmatrix}

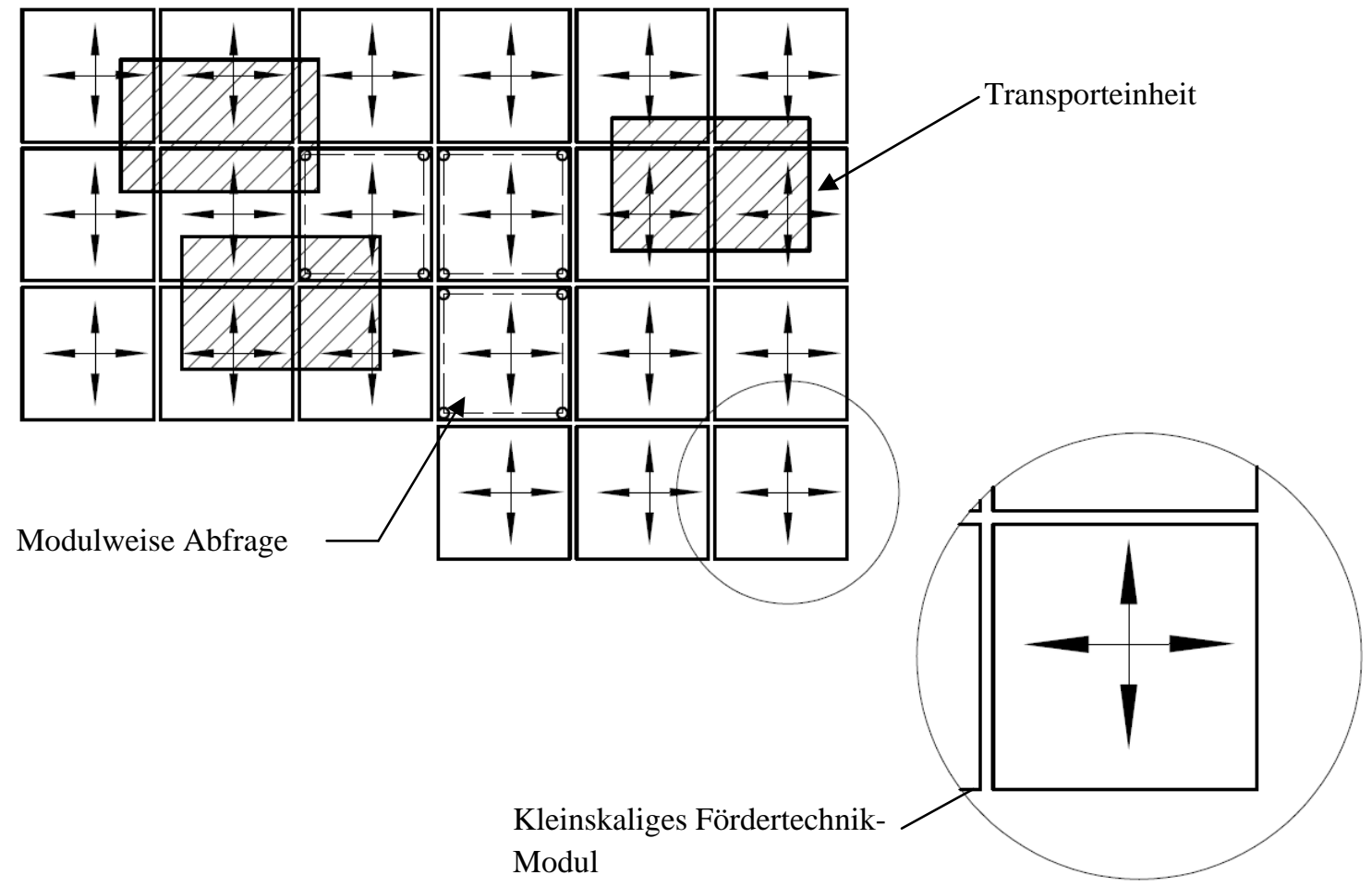

\section{Abbildung 1: Modulares System}

Um festzustellen, ob ein Modul mit z.B. quadratischer Grundfläche belegt ist, ist eine Erfassung an allen Kanten oder sogar ganzflächig ideal. Hier kann ein völlig neuer zukunftsweisender Ansatz zum tragen kommen, der es ermöglicht, photoelektrisch, also durch Lichtleitung, Positionen abzufragen. In /FAH 08/ wird ein Verfahren zum Dispensieren polymerer Lichtwellenleiter beschrieben. Hier werden optischer Polymere genutzt, um eine Bauteilintegration von Lichtwellenleitern (LWL) zu ermöglichen. Die LWL-Bahnen können in die Oberfläche des kleinskaligen Moduls direkt eingebracht werden und erlauben somit prinzipiell eine flächenhafte optische Erfassung.

Für die Energieversorgung der Antriebsmittel erscheint ein Schnittstellenkonzept geeignet, bei dem die Antriebsversorgung durch Kopplung von Element zu Element weitergeleitet wird, z.B. durch einen Energie-Bus. Berührungslose Systeme zur Energieübertragung sind bereits realisiert [BEU 01], bauen jedoch vergleichsweise groß und sind nachteilig im Wirkungsgrad.

Für die Informationsverarbeitung werden ebenso BUS-Systeme genutzt und weiterentwickelt werden. Denkbar ist hier eine drahtlose Kommunikation z. B. über WLAN oder ZigBee mit normiertem Sender und Empfänger an jedem zu steuernden Modul.

Mögliche Steuerungsmodelle werden z.Zt. untersucht in [OVE 09-01], [OVE09-02]. Im Rahmen dieser Untersuchungen, welche am Institut für Transport- und Automatisierungstechnik (ITA) der Leibniz Universität Hannover in Zusammenarbeit mit der Transnorm System GmbH durchgeführt werden, wird ein dreistufiges Steuerungsmodell mit einer übergeordneten Steuerungsebene für das gesamte Logistiksystem vorgeschlagen. Darunter befindet sich die Steuerungsebene für einzelne Materialflußfunktionalitäten wie Ausschleusungen oder Sortiervorgänge durch den Zusammenschluß von Logistikelementen. In der Subsystem-Ebene befindet sich unter der Steuerungsebene die Steuerungslogik, die dezentral auf die kleinskaligen Module verteilt ist. Die Zustände der Systeme und Subsysteme auf den Ebenen und deren zeitliche Änderungen werden durch 
Übergangsfunktionen beschrieben. Die Verknüpfung der Funktionen untereinander ist ein Teil dieser gesonderten Untersuchungen.

\section{Lösungsansätze auf der Basis existierender Technik}

Um die Komplexität der Lösungsfindung erfaßbar und überschaubar zu gestalten, bedient man sich zweckmäßiger Lösungsfindungsmethoden [PAH 93] und recherchiert in geeigneten Lösungskatalogen wie [KOL 98, EWA 75, LIN 08, ROT 00] . Zur systematischen Suche werden Ordnungsschemata eingesetzt, aus denen sich Lösungsansätze ableiten lassen und die die Verknüpfung der Einzellösungen erleichtern. Abb. 2 zeigt hierzu beispielhaft die Vorgehensweise.
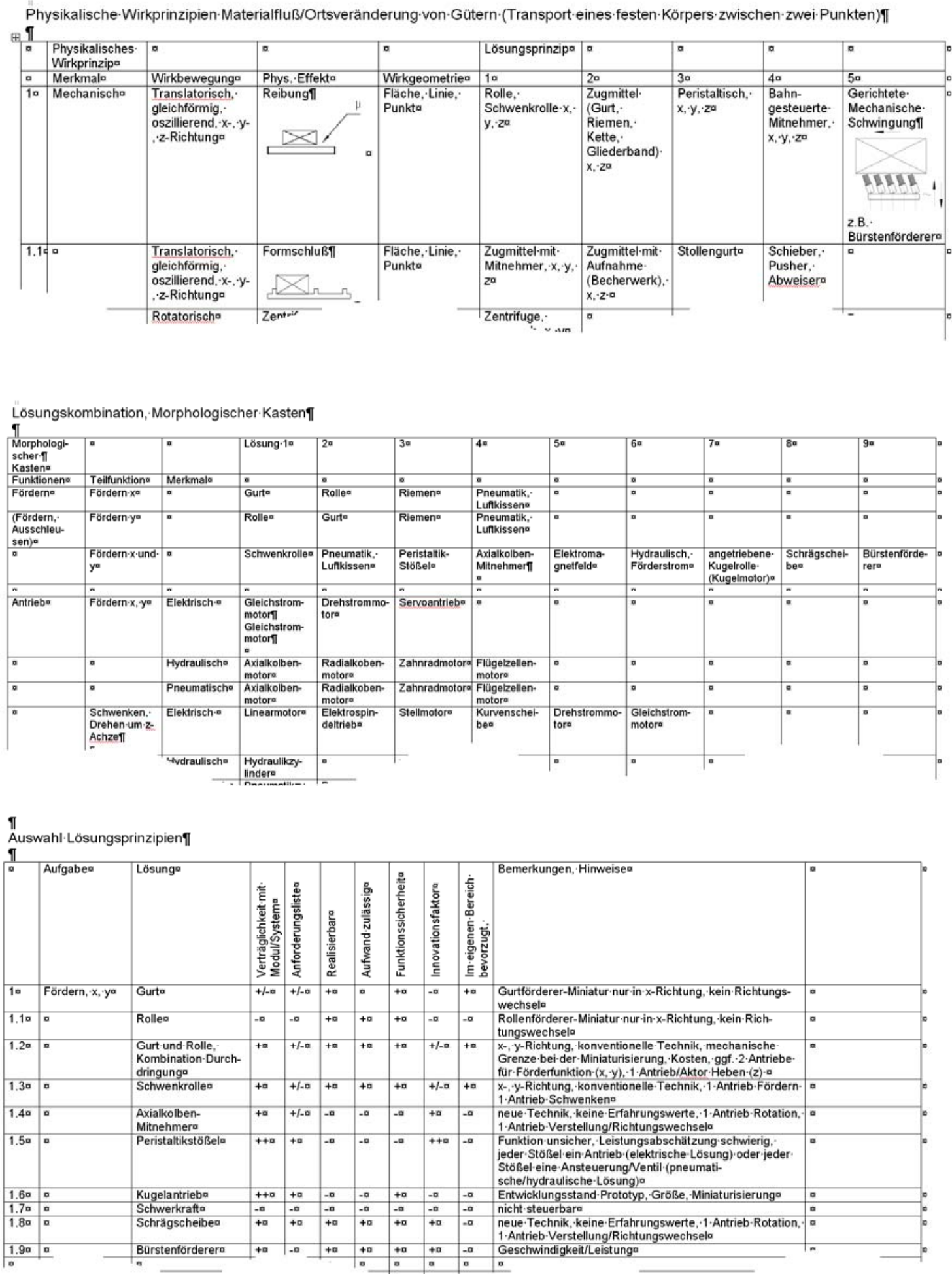


\section{Abbildung 2: Hilfsmittel zur systematischen Lösungsfindung, Tabellarische Ordnungsschemata}

Entsprechend der Ergebnisse dieser Lösungsfindung werden folgende Lösungsansätze als vielversprechend bewertet:

\section{Gurtförderer}

Es lassen sich kleinskalige Gurtförderer-Module miteinander koppeln. Je nach Größe der Module und der zu fördernden Transporteinheiten können sowohl in Längs- als auch in Querrichtung mehrere Module von einer Transporteinheit belegt werden.

Mit der Kopplung kleinskaliger Gurtförderer-Module ergibt sich nur in Förderrichtung eine Funktionalität (xRichtung). Sortieraufgaben und Ausschleusungen in y-Richtung sind so nicht möglich, es sei denn, die Module sind im Vergleich zur Größe der Transporteinheit sehr klein, so dass zwischen den Gurtfördermodulen Ausschleuseinrichtungen plaziert werden. Eine Ausführungsform ergibt sich in Form von HubRiemenausschleusern. Als Antrieb kommen je nach Kleinskaligkeit sowohl kleinere Drehstromantriebe als auch Gleichstromantriebe in Form von Rollenmotoren in Frage.

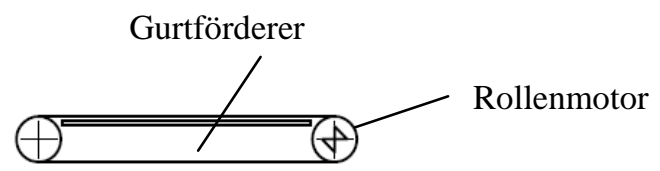

Abbildung 3: Prinzipskizze eine kleinskaligen Gurtförderers

\section{Rollenförderer}

Für die Funktionalität und Koppelbarkeit gilt ebenso wie für die Gurtförderermodule, dass Ausschleusungen und Funktionalitäten in y-Richtung, beispielsweise durch ergänzende Ausschleusmodule zwischen den Rollen, möglich sind. Der Ausschleuser hebt bei Bedarf zwischen den Rollen aus und fördert in y-Richtung. Zusammengefaßt ergibt diese Lösung ein bi-direktionales Modul, das den mechanischen Anforderungen entspricht. Als Antriebe kommen je nach Kleinskaligkeit sowohl kleinere Drehstromantriebe als auch Gleichstromantriebe in Form von Rollenmotoren in Frage.

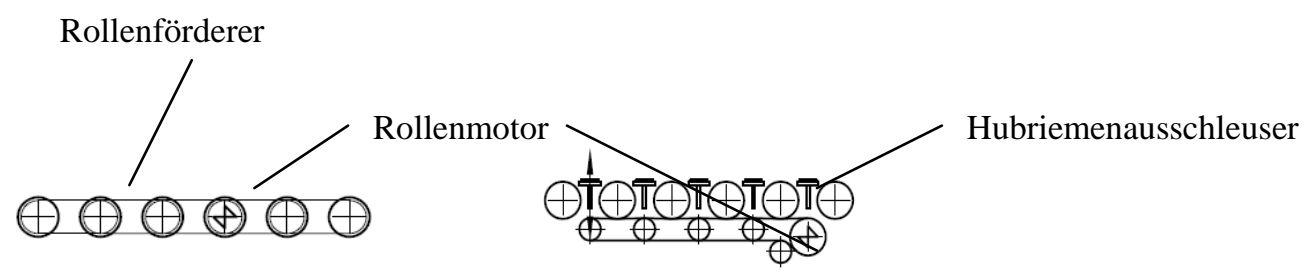

Abbildung 4: Prinzipskizze eines kleinskaligen Rollenförderers

\section{Schwenkrollensysteme}

Schwenrollensysteme sind idealerweise als Motorrolle ausgeführt, d.h. die Rolle hat das Antriebselement integriert. Vorzugsweise verwendet man einen regelbaren kleinen Gleichstromantrieb, der in einem Schwenkund Versorgungsmodul (Koppelmodul) mit gleicher Antriebsversorgung gelagert ist. Zum Fördern und Schwenken sind dann zwei möglichst gleichartig gestaltete Aktoren notwendig. So können n x n kleinskalige Schwenkrollenelemente zu einem Modul zusammengefaßt und verschaltet werden wie Abbildungen 5 und 6 dokumentieren. Derartige Elemente können ebenfalls ausschließlich mechanisch gekoppelt werden und konventionell, wie bei Schwenkrollenausschleusern zurzeit realisiert, einen Antrieb zum Fördern und zum Schwenken gemeinsam nutzen. Dieses reduziert die Investitionskosten und erleichtert die Realisierbarkeit erheblich.

Vorteil dieser Lösungen gegenüber den zuvor genannten ist die Sortierleistung. Gurt und Rollenmodule mit Ausschleusern haben ihre y-Funktionalität im Winkel von $90^{\circ}$ zur Hauptförderrichtung. Sortierfunktionen mit der Schwenkrolle sind deutlich Leistungsfähiger, da der Förderstrom hier in beliebigen Winkeln umgelenkt werden kann und somit aus dem kontinuierlichen Förderstrom verteilt werden kann.

Die entwickelten Fördermodule sind durch ihre Flexibilität für die Kopplung von kleinskaligen Fördersystemen geeignet. Ein Materialflußsystem, daß aus einem Verbund solcher Fördermodule besteht, ist z.Zt. kostenmäßig nicht konkurrenzfähig. Es konnte aber anhand eines Prototypen am Institut für Transport- und 
Automatisierungstechnik gezeigt werden, daß der Ansatz der Kopplung von kleinskaligen Fördersystemen mit integrierter Steuerungstechnik technisch umgesetzt werden kann und ein hohes Potential zur Steigerung der Flexibilität in der Intralogistik hat [vgl. OVE 09-01].

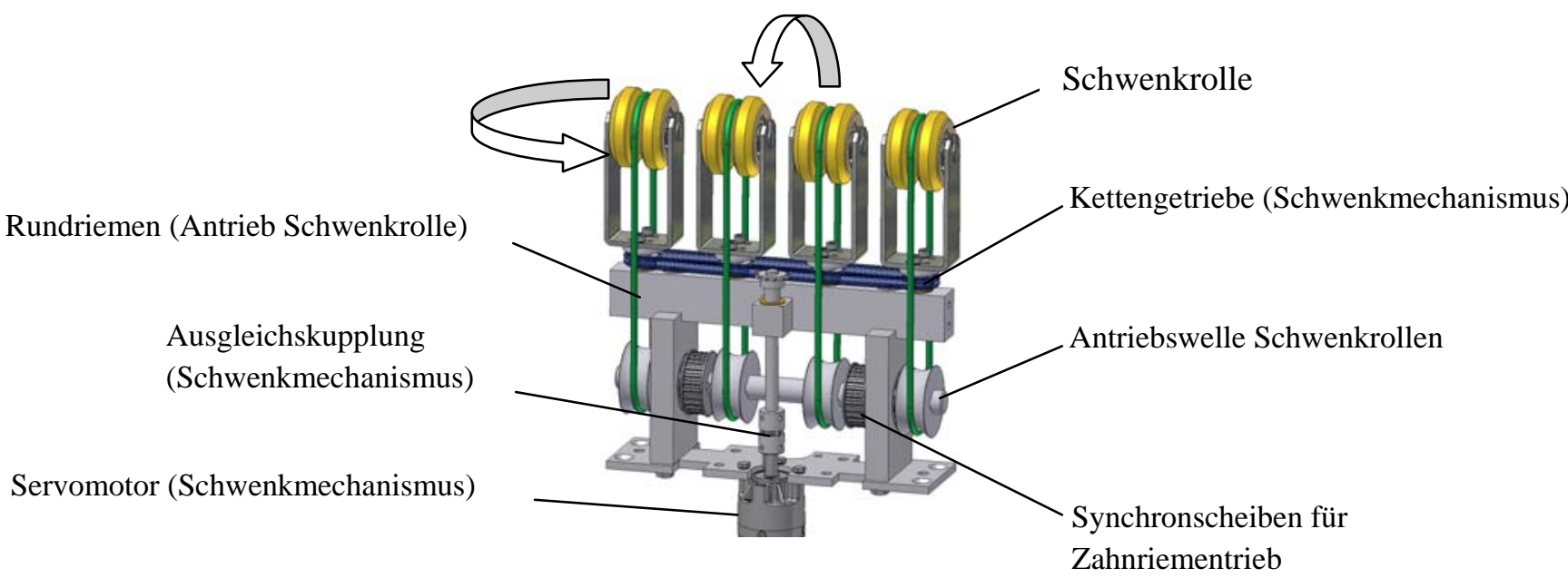

Kleinskaliges Fördermodul mit integrierter Sensorik
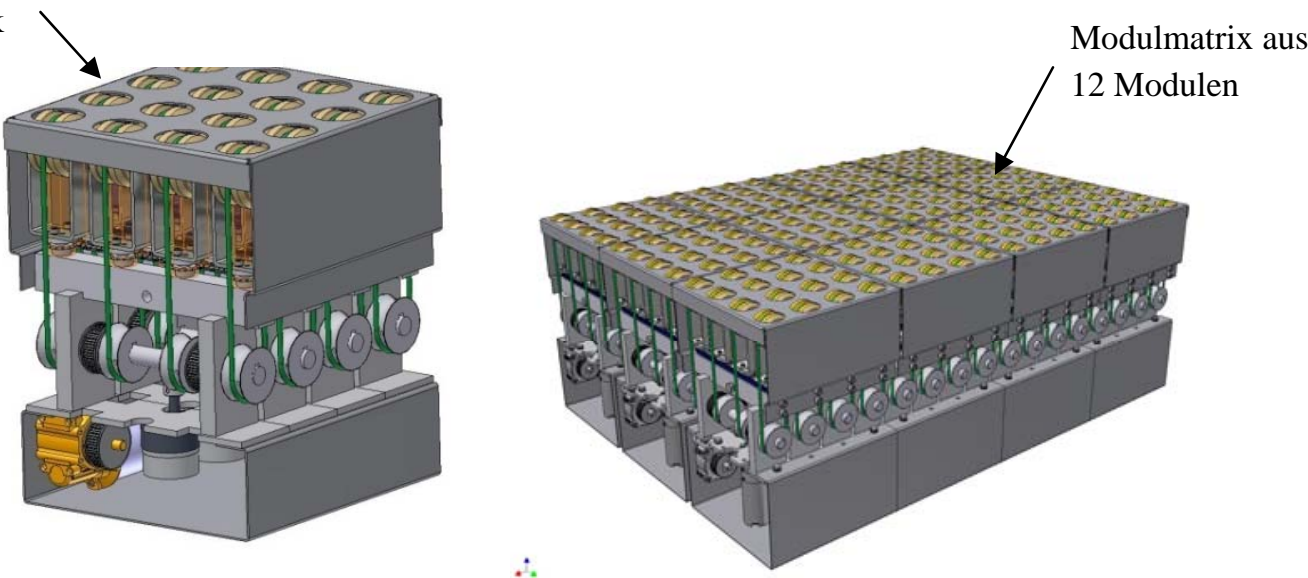

\section{Abbildung 5: Konzeptstudie auf der Basis von riemengetriebenen Schwenkrollen}

Versuche mit ersten Prototypen in Hinblick auf elektromotorische Schwenkrollensysteme am Institut für Transport- und Automatisierungstechnik (ITA) der Leibniz Universität Hannover zeigen ebenfalls, daß es möglich ist, eine elektromotorische Schwenk-/ Förderrolle mit konventionellen Mitteln zu entwickeln, welche die vorgegebenen Anforderungen an die Förder- und Schwenkgeschwindigkeit erfüllt. Entsprechende Ausführungsformen zeigt Abbildung 6 . 
DOI 10.2195/LJ_Ref_Overmeyer_de_2093_0909

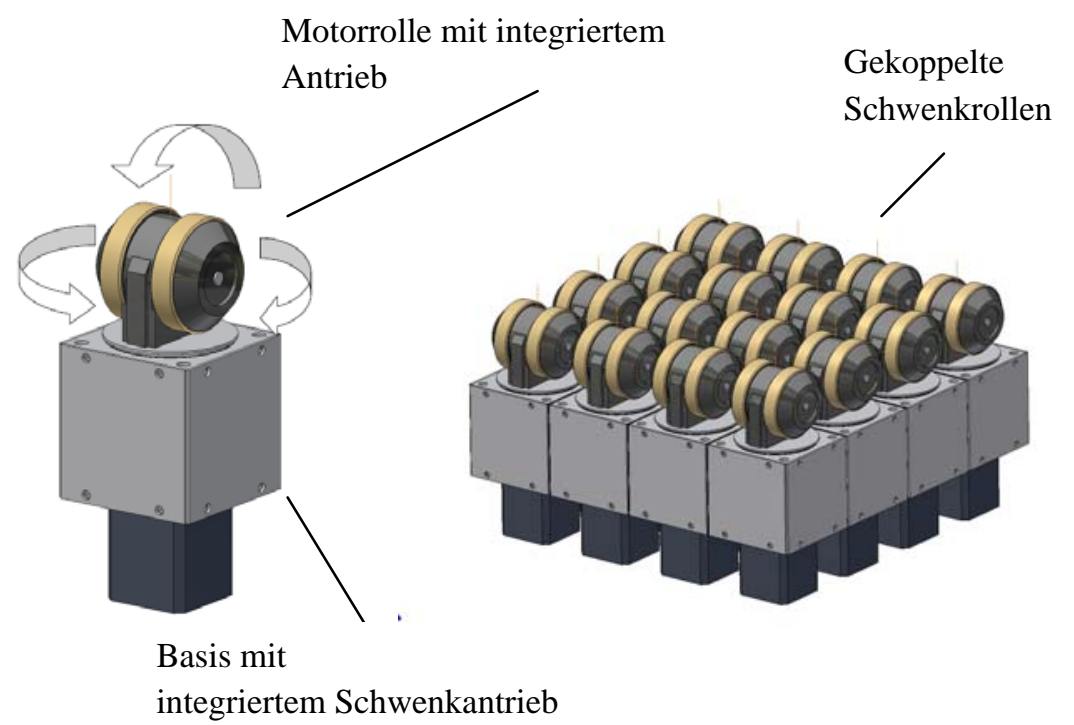

integriertem Schwenkantrieb

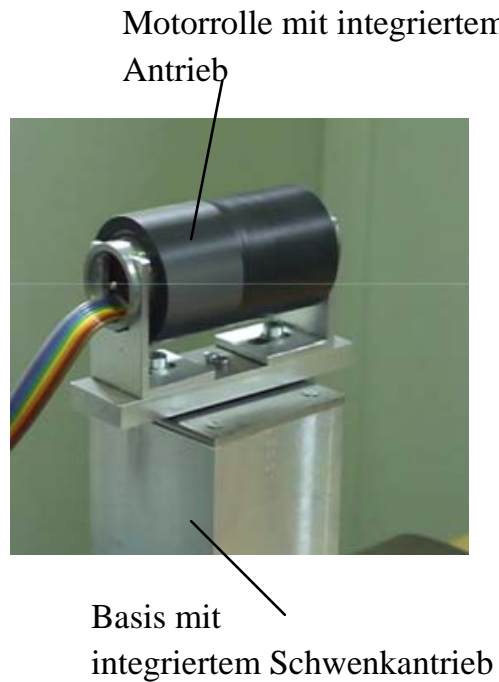

Prototyp Schwenkrolle, ITA 2008

Abbildung 6: Konzeptstudie auf der Basis von elektromotorisch angetriebenen Schwenkrollen 


\section{Neue Lösungsansätze für kleinskalige Module}

Im Folgenden sollen neue konstruktive Ansätze für die Entwicklung von kleinskaligen Modulen vorgestellt und anhand von ersten Konzeptstudien untersucht werden.

\section{Axialkolben-Mitnehmer}

Die Idee des Axial- oder Drehkolbenmitnehmers basiert auf der Grundkonstruktion einer Axialkolbenpumpe. Konzentrisch axial angeordnete Kolben oder Stößel rotieren in einer Scheibe um eine Achse und werden im Hub durch eine Schrägscheibe gesteuert. Die ausgefahrenen Stößel nehmen bei Rotation der Scheibe das Fordergut mit und tauchen nach dem Kontakt mit dem Fördergut wieder ab. Durch Verdrehung der Schrägscheibe kann bestimmt werden, bei welchem Drehwinkel die Kolben maximalen Hub haben und damit die Förderrichtung gesteuert werden. Eine entsprechende Ausführungsform zeigt Abbildung 7.

Bei diesem System handelt es sich um eine Neuentwicklung, es liegen daher noch keine praktischen Erfahrungen vor. Ein wesentlicher Gesichtspunkt in Bezug auf die Anwendbarkeit ist die Verträglichkeit mit üblichem Fördergut. Das System stellt durch die geringe Auflagefläche und die relativ weit verteilten Auflagepunkte besondere Anforderungen an die Fördergutbeschaffenheit. Der Boden muss sehr eben und fest sein. Es wären z.B. Kunststoffbehälter mit glattem Boden verwendbar. Die Bewegung der Stößel beschreibt einen Bogen. Das Fördergut wird im Berührungspunkt tangential zum Bogen bewegt. Auch Lösungen um die Geräuschentwicklung zu reduzieren sind zu betrachten.

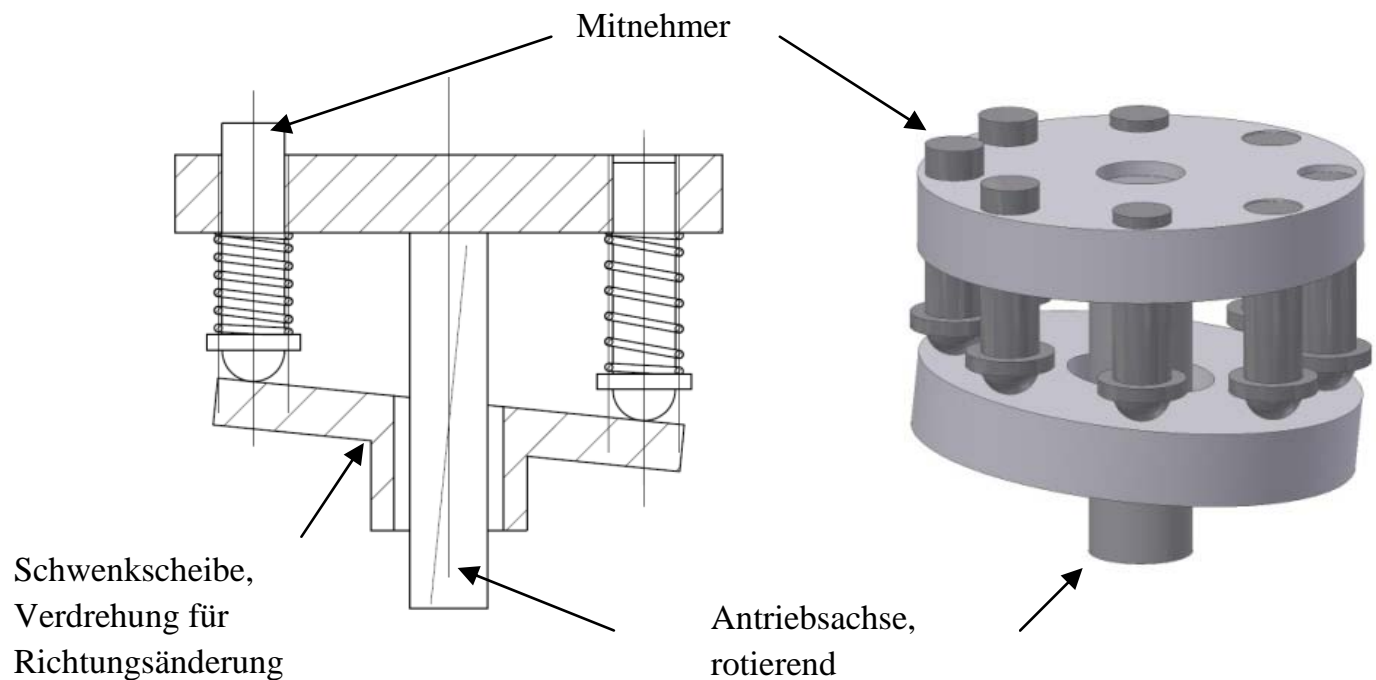

\section{Abbildung 7: Konzeptstudie eines Axialkolbenmitnehmers als modulares Förderelement}

\section{Schrägscheibe}

Die Schrägscheibe ist das vereinfachte Prinzip des dargestellten Axialkolbenmitnehmers. Eine zur Förderebene leicht geneigte rotationssymmetrische Scheibe berührt das Fördergut an ihrem höchsten, aus der Förderebene herausragenden Punkt und sorgt bei Rotation um die eigene Achse für Vortrieb. Bei Drehung der Scheibe um die z-Achse, die sich orthogonal zur Förderebene befindet, verlagert sich der höchste Punkt der Scheibe um die zAchse und damit die Richtung des Vortriebes. Somit ist auch eine Ausführung mit veränderbarer Förderrichtung denkbar. Eine entsprechende Ausführungsform zeigt Abbildung 8. 


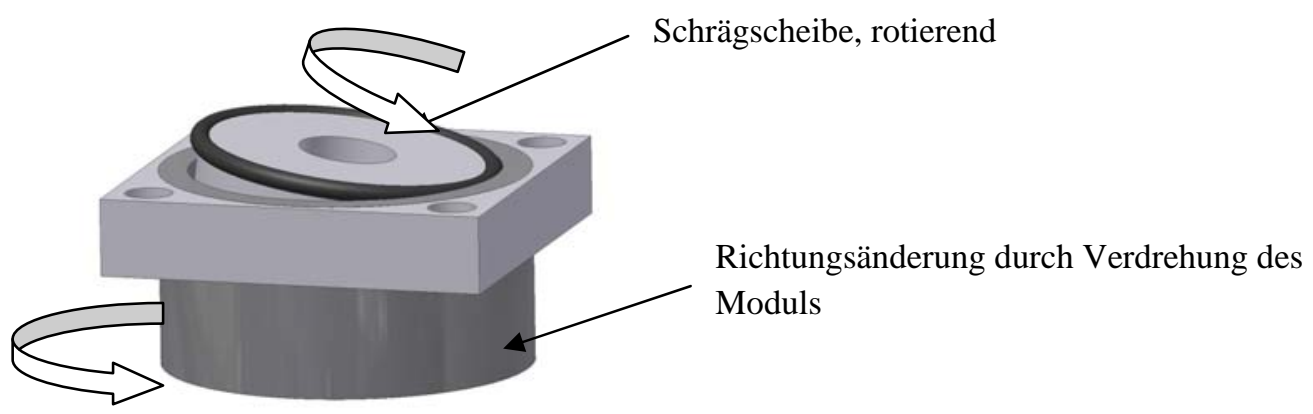

\section{Abbildung 8: Konzeptstudie einer Schrägscheibe als modulares Förderelement}

Durch den tangential bogenförmigen Vortrieb im Berührungspunkt sind unter Umständen Abweichungen von der idealen Förderrichtung möglich. Dieser Nachteil kann aber durch geschickte Anordnung der Elemente kompensiert werden, z.B. indem in x- und y-Richtung jeweils eine abwechselnde Anordnung von rechts bzw. links geneigten Elementen mit jeweils gegenläufigem Drehsinn erfolgt. Das Konzept der Schrägscheibe bietet die Möglichkeit, mehrere Schrägscheiben zu einem Modul zusammenzufassen und antriebstechnisch zu koppeln, um Antriebe einzusparen oder auf verfügbare, konventionell und größer bauende Antriebslösungen zurückgreifen zu können.

\section{Zusammenfassung und Ausblick}

Für die Lösung innerbetrieblicher Materialflußaufgaben bietet der Ansatz der koppelbaren, kleinskaligen Module ein erhebliches Rationalisierungspotential. Zum einen reduziert eine implementierte Intelligenz in Kombination mit dezentraler Steuerung und standardisierten Schnittstellen in den einzelnen Fördertechnik-Modulen den herkömmlichen Steuerungsaufwand. Die Module sind so einfach austauschbar oder an anderen Stellen des Systems einsetzbar. Die Kommunikation und Steuerung der Module untereinander wird z.Zt. gesondert am ITA im Rahmen des Projektes CogniLog untersucht [OVE 09-01], [OVE09-02].

Zum anderen gewinnt man durch kleinskalige Elemente an geometrischer Flexibilität. Die Gleichartigkeit der Module schafft produktionstechnische Vorteile. Voraussetzung für kleinskalige Gleichartigkeit ist idealerweise ein omnidirektional operierendes Fördertechnik-Modul, um Funktionalitäten wie Ausschleusen überall im System realisieren zu können. Einige fördetechnische Verfahren wie die Schwenkrollentechnik bieten hier bereits vielversprechende Ansätze, es werden aber auch neue Lösungen diskutiert.

Ein weiterer wichtiger Punkt bei der Verkleinerung der Module ist ein durch Größenreduktion eingeleiteter möglicher Technologiewechsel insbesondere im Bereich der Antriebstechnik.

Um das Potential der kleinskaligen Koppelbarkeit voll nutzen zu können, sind weitere Untersuchungen notwendig. Im Bereich der mechanischen Fördertechnik wird nach Prinzipien der Ortsveränderung gesucht, mit Hilfe derer sich kleinskalige, omnidirektionale Module realisieren lassen. Der Bereich der Steuerung für diese Aufgabe orientiert sich an dezentralen Intelligenzen und deren Kommunikation und im Bereich der Antriebstechnik wird der Einsatz mechatronischer Aktoren für Bewegungsvorgänge geprüft werden.

\section{Literatur}

[ARN 06] Arnold, D. (Hrsg.): Intralogistik - Potentiale, Perspektiven, Prognosen, Springer Verlag 2006 ISBN 3-540-29657-3

[BEU 01] Beumer, C.. Neuentwicklungen in der Logistik durch berührungslose Energieübertragung; Logistik für Unternehmen, 10/2001

[DEM 08] Dematic, Hersteller von Förderersystemen, modulares mechatronisches Stückgutfördersystem CL110, Katalogschrift

[DUB 05] Grothe, K.-H., Feldhusen, J. (Hrsg.): Dubbel, Taschenbuch für den Maschinenbau, Kapitel U5, Förderelemente und Fördersysteme für den innerbetrieblichen Materialfluß, Springer Verlag 2005 - ISBN 3-540-49714-5 
[EWA 75] Ewald, O.: Lösungssammlungen für das methodische Konstruieren; VDI-Verlag, Düsseldorf 1975 - ISBN 3-18-400310-8

[FAH 08] Fahlbusch, T.: Dispensieren polymerer Lichtwellenleiter, Dissertation Institut für Transport- und Automatisierungstechnik, Leibniz Universität Hannover 2008 - ISBN: 978-3-939026-53-2

[FLE 05] Fleisch, E.; Mattern, F. (Hrsg.): Das Internet der Dinge - Ubiquitous Computing und RFID in der Praxis, Springer Verlag 2005 - ISBN 3-540-24003-7

[HOM 05] Ten Hompel, M., Schmidt, T.: Warehouse Management, Springer Verlag 2005 - ISBN 3-54022509-9

[HOM 06] ten Hompel, M.: Dezentrale Steuerung für Materialflußsysteme am Beispiel von Stückgutförderund sortieranlagen, Logistics Journal 2006 - ISSN 1860-7977

[JOD 06] Jodin, D., ten Hompel, M.: Sortier- und Verteilsysteme, Grundlagen, Aufbau, Berechnung und Realisierung; Springer 2006 - ISBN 3-540-29070-2

[KOL 98] Koller, R., Kastrup, N.: Prinziplösungen zur Konstruktion technischer Produkte; Springer-Verlag 1998 - ISBN 3-540-63060-0

[LIN 08] Lindemann, U.: Physikalische Effektesammlung; Lehrstuhl für Produktentwicklung, TU München

[OVE 05] Overmeyer, L., Vogler, S.: RFID: Grundlagen und Potenziale. Logistics Journal, S. 1-12, ISSN 1860-5923 (2005).

[OVE 07] Overmeyer, L.: Innovative Gestaltung von Intralogistik durch Kopplung kleinskaliger Systeme, 16. Deutscher Materialfluss-Kongress; VDI-Berichte 1978, S. 171-179. Düsseldorf: VDI Verlag, 2007. - ISBN 978-3-18-091978-2

[OVE 09- Overmeyer, L.; Heiserich, G.; Falkenberg, S.; Jungk, A.: Automatische Konfiguration und 01] Optimierung von Materialflusssystemen durch kognitive Logistikmodule. In: VDI-Berichte Band 2066, 18. Deutscher Materialfluss-Kongress, München, 2009, S. 197-208.

[OVE 09- Overmeyer, L.; Heiserich, G.: Cognitive Transportation Modules for Adaptive Material Flow 02] Systems. In: Proc. Distributed Intelligent Systems and Technologies Workshop (DIST), St. Petersburg, 2009, S. 17-24.

[PAH 93] Pahl, G., Beitz, W.: Konstruktionslehre, Methoden und Anwendung, Springer-Verlag 1993 - ISBN 3-540-56194-3

[ROD 06] Roddeck, W.: Einführung in die Mechatronik, Teubner Verlag 2006 - ISBN 3-8351-0071-8

[ROT 00] Roth, K.: Konstruieren mit Konstruktionskatalogen, Springer-Verlag 2000 - ISBN 3-540-67142-0

[SEI 05] Seifert, W., RFID in der Logistik, Deutscher Verkehrsverlag 2005 - ISBN 3-871-54322-5

[TRA 08] Transnorm System GmbH, Hersteller von Fördersystemen, Moduline, modulares Fördertechniksystem, Katalogblätter

[WOL 07] Wolf-Kluthausen, H. (Hrsg.): Jahrbuch Logistik 2007, free beratung Gesellschaft für Kommunikation im Marketing GmbH, Unit Logistik, 2007 - ISBN 3-9809471-3-X 